\title{
Targeting nonsense-mediated mRNA decay in colorectal cancers with microsatellite instability
}

A'dem Bokhari ${ }^{1}$, Vincent Jonchere ${ }^{1}$, Anaïs Lagrange ${ }^{1}$, Romane Bertrand ${ }^{1}$, Magali Svrcek ${ }^{1,2}$, Laetitia Marisa (1) ${ }^{1,3}$, Olivier Buhard', Malorie Greene', Anastasia Demidova', Jieshuang Jia', Eric Adriaenssens ${ }^{5}$, Thierry Chassat ${ }^{6}$, Denis S. Biard', Jean-François Flejou ${ }^{1,2}$, Fabrice Lejeune ${ }^{4}$, Alex Duval ${ }^{1}$ and Ada Collura (i) ${ }^{1}$

\begin{abstract}
Nonsense-mediated mRNA decay (NMD) is responsible for the degradation of mRNAs with a premature termination codon (PTC). The role of this system in cancer is still quite poorly understood. In the present study, we evaluated the functional consequences of NMD activity in a subgroup of colorectal cancers (CRC) characterized by high levels of mRNAs with a PTC due to widespread instability in microsatellite sequences (MSI). In comparison to microsatellite stable (MSS) CRC, MSI CRC expressed increased levels of two critical activators of the NMD system, UPF1/2 and SMG1/ 6/7. Suppression of NMD activity led to the re-expression of dozens of PTC mRNAs. Amongst these, several encoded mutant proteins with putative deleterious activity against MSI tumorigenesis (e.g., HSP110DE9 chaperone mutant). Inhibition of NMD in vivo using amlexanox reduced MSI tumor growth, but not that of MSS tumors. These results suggest that inhibition of the oncogenic activity of NMD may be an effective strategy for the personalized treatment of MSI CRC.
\end{abstract}

\section{Introduction}

Microsatellite instability (MSI) colorectal cancers (CRC) are a subset of CRC characterized by defects in the DNA mismatch repair (MMR) system. MMR is a system for recognizing and repairing erroneous insertion, deletion, and misincorporation of bases that can arise during DNA replication and recombination, as well as for repairing some forms of DNA damage ${ }^{1}$. Inactivation of the MMR system is not in itself a direct transforming event, with additional genetic changes being necessary for MMRdeficient cells to become malignant. Mutations in human

\footnotetext{
Correspondence: Ada Collura (ada.collura@inserm.fr)

'Sorbonne Université, UPMC Univ Paris 06, INSERM, UMRS 938, SIRIC

CURAMUS, Equipe Instabilité des Microsatellites et Cancer, Equipe Labellisée par la Lique Contre le Cancer, Centre de Recherche Saint Antoine, Paris F-

75012, France

${ }^{2}$ AP-HP, Hôpital Saint-Antoine, Service d'Anatomie et Cytologie Pathologiques, Paris, France

Full list of author information is available at the end of the article.

Co-first authors A'dem Bokhari, Vincent Jonchere, Anaïs Lagrange
}

MMR proteins affect genomic stability, leading to widespread alterations in the length of microsatellite repeat sequences in the genome. The MSI phenotype was first observed in tumors from patients with Lynch syndrome (for review see ref. ${ }^{2}$ ), and later in sporadic cases of colon, gastric, and endometrial cancer. The highest incidence of MSI tumors occurs in epithelial cancers, but low frequencies of MSI have also been reported in many other cancer types ${ }^{3}$.

In MMR-deficient tumor cells, DNA polymerase slippage at coding sequences can induce frameshift mutations ( 1 or $2 \mathrm{bp}$ insertion/deletion) that results in the production of truncated proteins. Over the past 20 years, hundreds of different frameshift-truncating mutations have been reported in genes involved in various biological pathways such as cell cycle regulation (e.g., TGFBR2, IGFR2, TCF4, AXIN2, PTEN, RIZ), apoptosis (e.g., BAX, CASP5, BLC10, APAF1, FAS), DNA damage repair and DNA integrity checkpoint systems (e.g., $A T R, D N A-P K$,

\section{(c) The Author(s) 2018}

(c) (i) Open Access This article is licensed under a Creative Commons Attribution 4.0 International License, which permits use, sharing, adaptation, distribution and reproduction in any medium or format, as long as you give appropriate credit to the original author(s) and the source, provide a link to the Creative Commons license, and indicate if changes were made. The images or other third party material in this article are included in the article's Creative Commons license, unless indicated otherwise in a credit line to the material. If material is not included in the article's Creative Commons license and your intended use is not permitted by statutory regulation or exceeds the permitted use, you will need to obtain permission directly from the copyright holder. To view a copy of this license, visit http://creativecommons.org/licenses/by/4.0/. 
RAD50, MSH3, MSH6, MBD4, MLH3, BLM, CHK1) amongst others (for review see ref. ${ }^{2}$ ). By affecting microsatellites located proximally in the cDNA, most frameshift mutations result in the loss of tumor suppressor-related functions. In some cases, however, frameshift mutations can alter coding repeats that are located down-stream of important functional gene domains, or up-stream of other regulatory sites. In these latter contexts, such mutations are proposed to have a dominant negative effect, as for example with the Axin gene $^{4}$. In others, they are thought to be activating mutations, as proposed for the TCF-4 gene. Truncation mutations of TCF-4 in MSI cancers enhance the transactivating properties of this transcription factor by favoring the synthesis of isoforms that have lost their capacity to bind CtBP, a transcriptional repressor of the TCF/LEF family ${ }^{5}$.

Nonsense-mediated decay (NMD) is a ubiquitous surveillance mechanism that recognizes and degrades transcripts containing premature termination codon (PTC) ${ }^{6}$. A crucial aspect of the NMD pathway is the ability to distinguish normal termination codons from PTCs. The discrimination of PTC-containing mRNA in mammalian cells depends on the PTC position in the mRNA. mRNAs with a PTC located in the coding sequence excluding in the last exon, or more than $55 \mathrm{bp}$ up-stream of the last exon-exon junction, are recognized as NMD substrates ${ }^{7}$. In contrast, PTCs located down-stream of the last exon-exon junction are not degraded by the NMD system $^{8,9}$. The central component of the NMD pathway in mammalian cells is the protein UPF1. Activation of NMD leads to the transition of the SURF surveillance complex, consisting of the UPF1, SMG1, eRF1, and eRF3 factors, to the decay-inducing complex (DECID). The latter is formed following interaction of the SURF complex with UPF2, UPF3b, and an exon junction complex (EJC) downstream of the PTC. Remodeling of NMD complexes occurs following the phosphorylation of UPF1 by the protein kinase SMG1 ${ }^{10}$. Phosphorylated UPF1 proteins associate with the endonuclease proteins SMG5, SMG6, and $\mathrm{SMG7} 7^{11}$, and with general mRNA degradation factors such as POP2, leading to mRNA degradation ${ }^{12}$.

Approximately $30 \%$ of genetic disorders are due to nonsense or frameshift mutations that lead to PTC mRNAs encoding mutant proteins with no residual activity ${ }^{13,14}$. However, there are also examples where NMD degrades mutant proteins that have some residual activity. In such cases, NMD could have beneficial or detrimental effects depending on the mutation and on the disease ${ }^{15,16}$. In cancer, NMD can enhance the defects caused by frameshift mutations associated with partial loss of function in tumor suppressor genes ${ }^{15,17,18}$. Because of the high number of PTC-mRNAs generated by MSI in MMRdeficient neoplasms, NMD can halt the expression of many endogenous mutants, some of which may have a role in cancer development ${ }^{19-21}$. In MSI CRC, we and others have already highlighted the relevance of NMD in MSI-driven mutant mRNAs and in particular the important role of UPF1 in this process ${ }^{20}$. Despite its potential clinical relevance, the role of NMD in MSI tumorigenesis has not been investigated, possibly due to the lack of effective inhibitors of this system. In the present study, we used recently developed NMD inhibitors to target this system in MSI and MSS models of CRC. Our results highlight the important oncogenic impact of NMD on MSI-driven tumorigenesis and suggest that NMD inhibition may be a novel and effective strategy for the personalized treatment of MSI CRC.

\section{Results}

NMD factors are overexpressed in MMR-deficient primary colon tumors and NMD activity causes the decay of hundreds of MSI-driven PTC mRNAs

Expression levels for NMD-related factors (UPF1, UPF2, UPF3A, UPF3B, SMG1, SMG2, SMG6, and SMG7) were calculated from microarray-derived datasets of primary tumor samples $(n=40 \mathrm{MSI}, n=48 \mathrm{MSS})$ and matched normal colon samples $(n=95)$. The expression level of UPF1 and of other NMD-related factors was higher in primary MSI CRC vs MSS CRC (Fig. 1a). Consistent with its negative effect on NMD activity ${ }^{22}$, UPF3A expression was significantly down-regulated in MSI vs MSS primary tumors.

Exome sequencing of 30 MSI CRC from this cohort allowed us to identify MSI-driven somatic events within coding repeats (unpublished data). Significant downregulation of mutant mRNA was observed for genes with mutations in coding repeats that were located before the final exon (near last exon, NLE; $n=569$ ). This was not observed for mutant transcripts from genes harboring mutations contained within the final exon (last exon, LE; $n=98$ ) (Fig. $1 \mathrm{~b},{ }^{* * * *} p$-value $=1 \mathrm{E}-06$ ), indicating decay of these mRNA mutants in MSI tumor cells.

\section{Down-regulation of HSP110DE9 and other mutant mRNAs that encode cancer-related proteins with residual activities in MSI CRC}

From the 569 genes with NLE mutations, further studies were carried out on the 71 mutated MSI target mRNAs that displayed significant down-regulation (foldchange $<-0.5$ ) in MSI CRC (Supplementary Figure S1). These 71 genes included target genes for MSI with cancer-related functions (15 tumor suppressor-like genes and 16 oncogenes) and in which the frameshift mutated proteins may have retained complete or partial biological activity. Examples include the tumor suppressor genes WISP $^{23}, P A N K 1^{24}$ and SRFBP $1^{25}$, and the oncogene $J A K 1^{26,27}$ (Fig. 1c). 


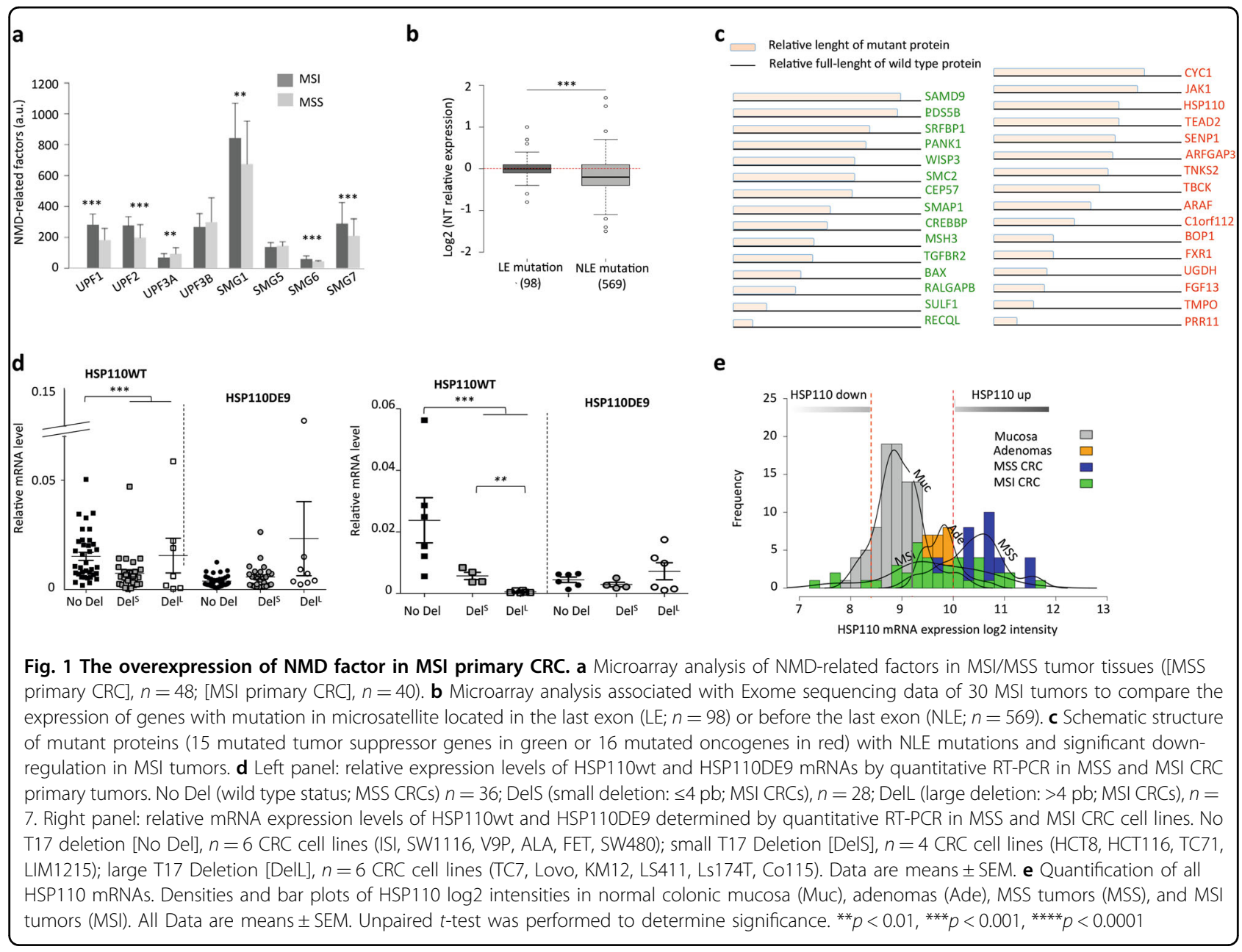

Of note, the HSP110 chaperone protein in MSI tumors is frequently altered by frameshift mutations. In these tumors, HSP110DE9 mRNA mutant is produced following bi-allelic deletions in the T17 noncoding DNA repeat of HSP110 intron $8^{28,29}$. Small deletions $(\leq 4 \mathrm{bp})$ of T17 do not induce aberrant splicing of exon 9 , whereas large deletions $(>4 \mathrm{bp}$ ) induce aberrant exon 9 skipping of the HSP110 gene. This leads to an HSP110DE9 mRNA containing a PTC before the last exon, which is therefore sensitive to mRNA decay. HSP110 was found in the list of MSI target genes with significant mRNA downregulation in MSI CRC (Fig. 1c). Using quantitative RT-PCR, the expression of HSP110 wild type mRNA was found to be significantly lower in MSI CRC cell lines and primary tumors displaying large T17 deletions (Fig. 1d). In contrast, the expression of HSP110DE9 mutant transcript was not increased in these samples. Micro-array analyses performed on a larger series of CRC confirmed the lower HSP110 wild type mRNA level in MSI CRC compared to MSS CRC (Fig. 1e). Similar to other frameshift mutant mRNAs encoding aberrant oncoproteins with residual biological activity (Fig. 1c), we postulate that HSP110DE9 produces a dominant negative isoform of HSP110 that is strongly degraded by NMD in MSI CRC cells (Fig. 1c-e).

\section{Inhibition of NMD in colorectal cancer cells and in mouse models can be achieved successfully using commercial NMD inhibitors}

CRC cell lines were treated with two commercially available NMD inhibitors: (i) amlexanox, for which the exact mechanism of action is still unknown ${ }^{30}$, and (ii) NMDI-1, which inhibits the dephosphorylation of UPF1 and thereby keeps it in a non-active state ${ }^{31}$. Two controls were used: an siRNA against the key NMD activator UPF1, and the protein synthesis inhibitor cycloheximide (CHX). As shown in Fig. 2a, treatment with these four agents all resulted in significant re-expression of the exogenous HSP110DE9 transcript following transfection of MSS SW480 CRC cell lines with a plasmid specially designed for this purpose.

A panel of CRC cell lines was also used to study the differential decay of PTC-mRNAs resulting from MSIdriven mutations. Treatment with $\mathrm{CHX}$ increased the expression of mutant PTC-mRNAs derived from MSI 


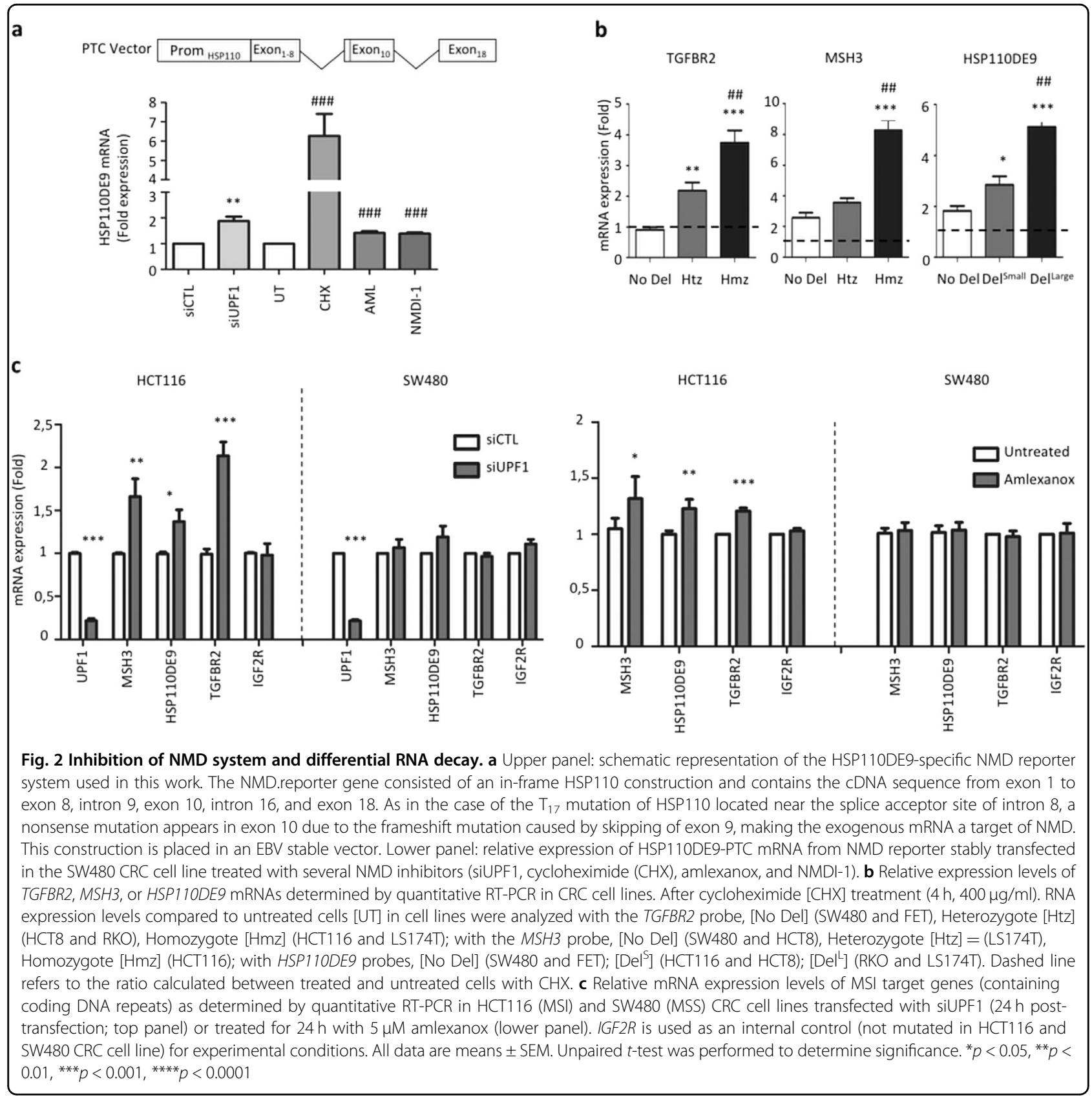

target genes (e.g., TGFBR2, MSH3, or HSP110) in MSI vs MSS CRC cell lines (Fig. 2b). Re-expression of HSP110DE9 mRNA was demonstrated using siRNA targeted to the major NMD factor UPF1, and following amlexanox treatment of the MSI CRC cell line HCT116. UPF1 depletion and amlexanox treatment also led to significant increases in the expression of other mutated PTC-containing transcripts (TGFBR2, MSH3), but not of a wild type mRNA control (IGFR2). As expected, the levels of endogenous mutant mRNA in the MSS CRC cell line SW480 were not altered following inhibition of NMD (Fig. 2c).
Inhibition of NMD leads to decreased MSI CRC cell proliferation and has an antitumor effect on MSI tumor xenografts

The above results suggest that NMD is responsible for the decay of hundreds of PTC-mRNAs in MSI CRC cells, including several that encode cancer-related mutant proteins with residual activity such as HSP110DE9. We next compared the functional consequences of NMD inhibition between MSI and MSS cell lines. These are characterized by relatively high and low numbers of PTCmRNAs, respectively. Inhibition of UPF1 caused 


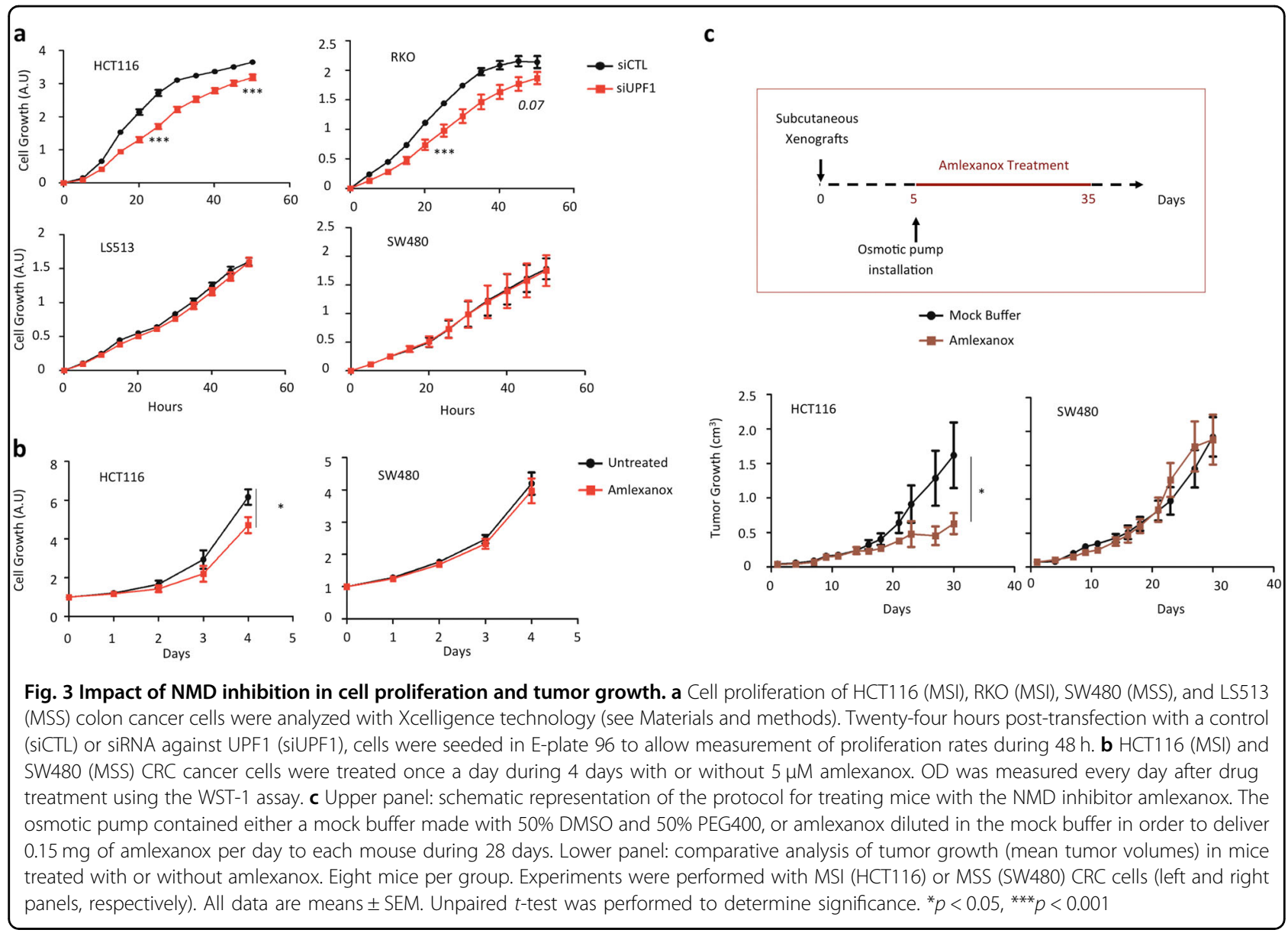

decreased cell proliferation in the MSI CRC cell lines (HCT116 and RKO), whereas no effect was seen in the MSS cell lines (LS513 and SW480) (Fig. 3a and Fig. S2). Similarly, amlexanox treatment decreased the cell proliferation rate in HCT116 cells (MSI) but not in SW480 cells (MSS, Fig. 3b).

NMD inhibition using amlexanox led to a significant antitumor effect in xenografts of MSI CRC cells (HCT116) grown in nude mice when compared to treatment with control buffer only (Fig. 3c). However, this effect was not observed in nude mice xenografted with MSS CRC cells (SW480).

\section{Discussion}

In this study, we report the specific overexpression of essential NMD factors such as UPF1/2 and SMG1/6/7 in MSI primary CRC compared to MSS primary CRC. Furthermore, NMD inhibition was shown to adversely affect MSI cell proliferation but not MSS cell proliferation in vitro, and to inhibit MSI tumor growth but not MSS tumor growth in a xenograft model. From a mechanistic standpoint, these results suggest that NMD activity has an oncogenic impact on tumorigenesis in MSI CRC. We have identified a role for NMD in the down-regulation of confirmed or putative toxic mutant proteins that derive from PTC-mRNAs, such as HSP110DE9 ${ }^{28,29}$ and others. Our data also show that NMD inhibition using amlexanox, a known inhibitor of $\mathrm{NMD}^{30}$, was also efficient in a mouse xenograft model. These in vivo results suggest that NMD could be an interesting target for inhibition in the treatment of patients with MSI colon cancer or other MMR-deficient primary tumors.

In previous publications, we and others have proposed that NMD may play a role during MSI tumorigenesis ${ }^{20,32}$. Nevertheless, this putative role was poorly understood and thought to be quite complex depending on the gene function for the mutation targeted by NMD factors. Previous exome sequencing analysis of 30 MSI primary cancers performed by our group allowed us to comprehensively identify all indel-mutations that lead to PTC formation and are targeted by NMD. Amongst the 71 mutated MSI target genes in which the PTC-mRNAs were expected to be down-regulated by NMD, we identified 15 aberrant transcripts encoding mutant proteins with putative retained tumor suppressor functions, including PDS5B, WISP3, and PANK1. The PDS5B mutant protein, 


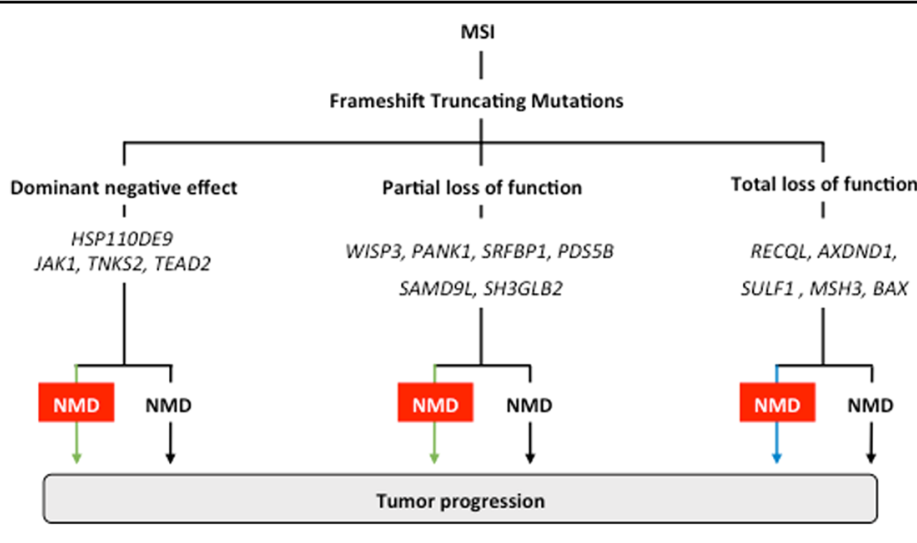

Fig. 4 Schematic representation of the role of NMD in MSI tumors and the expected therapeutic benefit of NMD blockade in this tumor model. Numerous MSI-driven mutant PTC-mRNAs processed by NMD are generated due to frameshift-truncating mutations in coding DNA microsatellites in MMR-deficient colon tumors. These mutations usually result in loss of function effects and they inactivate tumor suppressor genes (e.g., BAX, MSH3...). Although the corresponding mutant PTC-containing mRNAs are degraded by NMD or not it is of poor functional significance when the mutant proteins have totally lost their function and therefore do not have residual biological activity. In contrast, it is expected that inhibiting NMD could be deleterious for MSI cancer cells when mutant mRNAs encode for (i) tumor-suppressor mutant proteins that have retained some residual activity (notably, partial loss of function is expected when the truncation occurs down-stream of important functional domains in the protein), or (ii) dominant negative mutant proteins like HSP110DE9

for example, retains all the regions and residues involved in the recruitment and release of cohesin and other partners during mitosis, suggesting it has conserved tumor suppressor activity in MSI cancer cells ${ }^{33}$. Moreover, we identified 16 aberrant transcripts that encoded oncoproteins, including HSP110, JAK1, ARAF, and TNKS2. As previously reported, HSP110DE9 protein has dominant negative activity and its aberrant mRNA is targeted by NMD. HSP110DE9 interacts with wild type HSP110, thereby strongly inhibiting its oncogenic function $^{28,29,34}$. Another putative dominant negative mutation affects the ARAF proto-oncogene in MSI CRC cells. The MSI-driven frameshift mutation of $A R A F$ results in a truncated protein due to deletion of 293 aa. This mutant is closely related to the DA-Raf1 dominant-negative mutant, which lacks the kinase domain and is reportedly an antagonist of the Ras-Erk pathway ${ }^{35}$. Therefore, several mechanisms are likely to underpin the oncogenic effects of NMD in MSI CRC and these will require further investigation in future studies. Figure 4 shows the putative role of NMD in MSI tumors and how a therapeutic benefit from NMD inhibition could be expected in this model.

A major finding of this work was the demonstration of efficient inhibition of NMD activity using Amlexanox. Other pharmacological agents have previously been used to inhibit NMD, including cycloheximide ${ }^{36}$, emetine ${ }^{37}$, puromycin ${ }^{38}$, pateamine $\mathrm{A}^{39}$, caffeine, wortmannin, and NMDI-1 ${ }^{15,40,31}$. However, these are not suitable for clinical use because of their toxicity or weak activity. In contrast, the NMD inhibitor amlexanox is already being used to treat patients with recurrent aphthous stomatitis ${ }^{41}$. We demonstrated here that treatment with amlexanox resulted in significant re-expression of several PTC mRNAs encoding HSP110DE9 and also other toxic mutant proteins. Probably as a consequence of this, MSI cancer cells are particularly sensitive to NMD inhibition. Amlexanox treatment led to significant and specific inhibition of the growth of MSI tumor xenografts in vivo, while showing no apparent toxicity in mice or in humans treated for aphthous ulcers ${ }^{42}$. However, it should be noted that amlexanox has been reported to inhibit TBK1 and IKK- $\varepsilon$ protein kinases in mice ${ }^{43}$ and could therefore have other effects unrelated to NMD inhibition, as found here. Moreover, although amlexanox is already in clinical use, it is clear that it was not a strong inhibitor of NMD in our hands. In future studies, we plan to use other agents that could be more powerful inhibitors of NMD in tumor cells. Interestingly, such agents could also be of therapeutic interest since they are expected to enhance $\mathrm{T}$ cell antitumor immunity in MSI CRC by favoring the presentation of numerous MSI-driven aberrant neoantigens at the surface of tumor cells. Whether NMD inhibition could be used synergistically with immune checkpoint inhibitors that are currently being trialed in patients with MSI cancer remains to be determined.

\section{Materials and methods}

\section{CRC cell lines and clinical tumor samples}

CRC cell lines were obtained from the American Type Culture Collection and were grown in DMEM containing $10 \%$ fetal bovine serum and antibiotics. They were free of mycoplasma. Tissue samples of primary tumor and normal colon were obtained from patients undergoing 
surgery for CRC (Hôpital Saint-Antoine, Paris, France). The MSI status of tumors was assessed as reported previously $^{44}$.

\section{Analysis of HSP110 T17 deletion and real-time quantitative RT-PCR}

The QIAmp DNA Mini Kit (Qiagen) was used to extract DNA. The deletion status of the T17 repeat in HSP110 was determined as previously described ${ }^{45}$. Total RNA was obtained using the RNeasy Mini kit (Qiagen). The integrity of RNA (RIN) from all primary tumor samples was assessed on a 2100 Bioanalyzer using the RNA 6000 Nano LabChip kit (Agilent). Only those with RIN of $>5$ were evaluated further. Complementary DNA was made using a High Capacity cDNA reverse transcription kit (Applied Biosystems) and the Applied SDS Biosystems analysis software used for quantitative RT-PCR. HSP110wt and $H S P 110 D E 9$ transcript levels were calculated relative to RPLP0 ubiquitous RNA, while TGFBR2, MSH3, BAX, $I G F 2 R$, and GAPDH transcript levels were calculated relative to $18 \mathrm{~S}$ ubiquitous RNA. The primers and probes for HSP110wt and HSP110DE9 were reported previously ${ }^{45}$. Primers and internal probes for TGFBR2, MSH3, BAX, IGF2R, GAPDH, 18S, and RPLPO were those recommended by Applied Biosystems (TaqMan gene expression assays). The cycling conditions for PCR consisted of an initial denaturation step at $95^{\circ} \mathrm{C}(10 \mathrm{~min}$ duration) followed by 40 cycles of $95^{\circ} \mathrm{C}(15 \mathrm{~s})$ and $60^{\circ} \mathrm{C}$ (1 min).

\section{In vitro treatments with cycloheximide, NMDI-1 and amlexanox}

Cells were grown in 6-well plates $\left(2 \times 10^{5}\right.$ cells seeded per well) in DMEM media. Cycloheximide (SigmaAldrich) was added at $400 \mu \mathrm{g} \cdot \mathrm{ml}^{-1}$ for $4 \mathrm{~h}$, amlexanox (Sequoia Research Products) at $5 \mu \mathrm{M}$ for $24 \mathrm{~h}$, or NMDI-1 (S.E. Velu Laboratory) at $2.5 \mu \mathrm{M}$. Cells were then harvested and the RNA extracted using RNeasy Mini Kit $\left(\right.$ Qiagen $\left.^{\circledR}\right)$.

\section{Transfection with siRNA}

HCT116 CRC cells were grown in 6-well plates $\left(1 \times 10^{5}\right.$ cells seeded per well) and then transfected with siRNA $(50 \mathrm{nM})$ targeted against UPF1 or else with non-specific siRNA (DharmaFECT 1, Thermo Fisher). siRNA targeted against GAPDH was used as the control. Cells were harvested for total RNA extraction at $48 \mathrm{~h}$ after transfection, with all experiments done in triplicate.

\section{Cell proliferation}

The reagent WST-1 (SIGMA $\left.{ }^{\circledast}\right)$ was used to quantify cell proliferation. Cells $\left(2 \times 10^{4}\right.$ per well $)$ were seeded into 24 well plates and grown in $2 \mathrm{~mL}$ of supplemented DMEM medium. They were treated once each day with amlexanox for 4 days at concentrations of 5 or $25 \mu \mathrm{M}$. For the cell proliferation assay, WST-1 reagent was added at each time point and incubation was for $4 \mathrm{~h}$ at $37^{\circ} \mathrm{C}$. Absorbance measurements were made at $450 \mathrm{~nm}$ and the reference wavelength used was $750 \mathrm{~nm}$. Rates of cell proliferation were evaluated using XCelligence $\left(\mathrm{OZYME}^{\oplus}\right.$ and ACEA Biosciences ${ }^{\circledR}$ ). Following transfection with siUPF1, cells were plated in E-plate 96 containing gold sensor arrays (electrodes). Measurement of impedance by the sensor electrodes allows any physiological changes to cells to be monitored.

\section{Whole-exome sequencing}

A series of 30 MSI CRC and paired adjacent normal mucosa were previously sequenced for mutations in microsatellite sequences (https://www.ebi.ac.uk/ega/ home; Accession No. EGAS00001002477). The position of mutations in the transcripts was based on RefSeq hg19 annotation. mRNA expression in these tumors was also assessed using microarrays as previously described.

\section{Measurement of HSP110 and NMD factor mRNA expression}

A series of MSI $(n=40)$ and MSS $(n=48)$ CRC, as well as normal colonic mucosa $(n=42)$ were evaluated for mRNA expression using Affymetrix U133Plus chips ${ }^{46}$ (data partially contained within the GSE33582 data set). Additional adenoma and normal mucosa samples were included from the GSE8671 and GSE4183 data sets. Data was normalized (Robust Multi-array Average normalization, $R$ package affy) and associations with variables were estimated using ANOVA or $t$-test (R package stats). Comparison of NMD factor expression between MSI and MSS tumors was made using $t$-test and corrected for multiple testing ( $R$ package limma).

\section{In vivo effect of amlexanox}

In the xenograft experiments, approximately $10^{7}$ cells (SW480 or HCT116 CRC cell lines) were injected subcutaneously into the right flank of 5-week old female nude mice (Charles River Laboratories). After the tumor had grown to about $4 \mathrm{~mm}$, an osmotic pump was placed subcutaneously on the left flank of each animal. These contained either a $50 \% \mathrm{DMSO} / 50 \%$ PEG400 buffer, or the same buffer containing amlexanox. Tumor growth was determined by caliper measurement 3 times per week for 30 days and blinded to the treatment status. Mice were treated in accordance with the ethical regulations of the French Ministry of Research and Technology.

\section{HSP110DE9-NMD assays}

The HSP110DE9-specific NMD-target vector was made by cloning into an EVB plasmid an insert containing the HSP110 cDNA sequence: exons $1-8$, intron 8 , exon 10 , 
intron 16, and exon 18. This was under the control of the HSP110 promoter. Both HCT116 and SW480 cells were transfected with this HSP110DE9-specific NMD-target vector using Lipofectamine (Invitrogen). Cells were treated with amlexanox ( 5 or $25 \mu \mathrm{M})$ at $24 \mathrm{~h}$ after the start of transfection and $24 \mathrm{~h}$ later the cells were harvested for analysis of RNA expression.

\section{Acknowledgements}

We thank Dr. Barry lacopetta for critical reading of the manuscript. This work was supported by grants from the Institut National du Cancer (INCa). A.D.'s group has the label "La Ligue Contre le Cancer". The present work has benefited from the animal facility of Saint-Antoine Research Center (PHEA, Mrs Tatiana Ledent) and the core facility of UMS30-LUMIC, CISA (Cytometry and Imagery Saint Antoine, Mrs Annie Munier), Sorbonne University, UPMC Univ Paris 06, INSERM, Saint-Antoine (CRSA), F-75012 Paris, France.

\section{Author details}

'Sorbonne Université, UPMC Univ Paris 06, INSERM, UMRS 938, SIRIC CURAMUS, Equipe Instabilité des Microsatellites et Cancer, Equipe Labellisée par la Ligue Contre le Cancer, Centre de Recherche Saint Antoine, Paris F75012, France. ${ }^{2}$ AP-HP, Hôpital Saint-Antoine, Service d'Anatomie et Cytologie Pathologiques, Paris, France. ${ }^{3}$ Programme "Cartes d'Identité des Tumeurs", Ligue Nationale Contre le Cancer, Paris, France. ${ }^{4}$ Univ. Lille, CNRS, Institut Pasteur de Lille, UMR 8161-M3T-Mechanisms of Tumorigenesis and Target Therapies, F-59000 Lille, France. ${ }^{5}$ INSERM U908, Cell Plasticity and Cancer, University of Lille, 59655 Villeneuve d'Asca, France. ${ }^{6}$ Pasteur de Lille-PLEHTA (Plateforme d'expérimentation et de Haute Technologie Animale), 59019 Lille, France. ${ }^{7}$ Centre d'Etude Atomique, Direction des Sciences du Vivant, Institut des Maladies Emergentes et des Thérapies Innovantes, Service d'Etude des Prions et des Infections Atypiques, Fontenay-aux-Roses, France

\section{Conflict of interest}

The authors declare that they have no conflict of interest.

\section{Publisher's note}

Springer Nature remains neutral with regard to jurisdictional claims in published maps and institutional affiliations.

Supplementary Information accompanies this paper at (https://doi.org/ 10.1038/s41389-018-0079-x).

Received: 9 April 2018 Revised: 6 June 2018 Accepted: 24 July 2018 Published online: 19 September 2018

\section{References}

1. Larrea, A. A., Lujan, S. A. \& Kunkel, T. A. SnapShot: DNA mismatch repair. Cell 141, 730e1 (2010)

2. Duval, A. \& Hamelin, R. Mutations at coding repeat sequences in mismatch repair-deficient human cancers: toward a new concept of target genes for instability. Cancer Res. 62, 2447-2454 (2002).

3. Hause, R. J., Pritchard, C. C., Shendure, J. \& Salipante, S. J. Classification and characterization of microsatellite instability across 18 cancer types. Nat. Med. 22, 1342-1350 (2016).

4. Liu, W. et al. Mutations in AXIN2 cause colorectal cancer with defective mismatch repair by activating beta-catenin/TCF signalling. Nat. Genet. 26, 146-147 (2000).

5. Duval, A. et al. The human T-cell transcription factor-4 gene: structure, extensive characterization of alternative splicings, and mutational analysis in colorectal cancer cell lines. Cancer Res. 60, 3872-3879 (2000).

6. Popp, M. W. \& Maquat, L. E. Organizing principles of mammalian nonsensemediated mRNA decay. Annu. Rev. Genet. 47, 139-165 (2013).

7. Nagy, E. \& Maquat, L. E. A rule for termination-codon position within introncontaining genes: when nonsense affects RNA abundance. Trends Biochem. Sci. 23, 198-199 (1998).
8. Byers, P. H. Killing the messenger: new insights into nonsense-mediated mRNA decay. J. Clin. Invest. 109, 3-6 (2002).

9. Lykke-Andersen, J., Shu, M. D. \& Steitz, J. A. Communication of the position of exon-exon junctions to the mRNA surveillance machinery by the protein RNPS1. Science 293, 1836-1839 (2001).

10. Yamashita, A. et al. SMG-8 and SMG-9, two novel subunits of the SMG-1 complex, regulate remodeling of the mRNA surveillance complex during nonsense-mediated mRNA decay. Genes Dev. 23, 1091-1105 (2009).

11. Chakrabarti, S., Bonneau, F., Schussler, S., Eppinger, E. \& Conti, E. Phosphodependent and phospho-independent interactions of the helicase UPF1 with the NMD factors SMG5-SMG7 and SMG6. Nucleic Acids Res. 42, 9447-9460 (2014).

12. Schoenberg, D. R. \& Maquat, L. E. Regulation of cytoplasmic mRNA decay. Nat. Rev. Genet. 13, 246-259 (2012).

13. Frischmeyer, P. A. \& Dietz, H. C. Nonsense-mediated mRNA decay in health and disease. Hum. Mol. Genet. 8, 1893-1900 (1999).

14. Keeling, K. M. \& Bedwell, D. M. Suppression of nonsense mutations as a therapeutic approach to treat genetic diseases. Wiley Interdiscip. Rev. RNA 2, 837-852 (2011).

15. Usuki, F. et al. Inhibition of nonsense-mediated mRNA decay rescues the phenotype in Ullrich's disease. Ann. Neurol. 55, 740-744 (2004).

16. Usuki, F. et al. Specific inhibition of nonsense-mediated mRNA decay components, SMG-1 or Upf1, rescues the phenotype of Ullrich disease fibroblasts. Mol. Ther. 14, 351-360 (2006).

17. Bhuvanagiri, M., Schlitter, A. M., Hentze, M. W. \& Kulozik, A. E. NMD: RNA biology meets human genetic medicine. Biochem. J. 430, 365-377 (2010).

18. Popp, M. W. \& Maquat, L. E. Nonsense-mediated mRNA decay and cancer. Curr. Opin. Genet. Dev. 48, 44-50 (2018).

19. Chan, W. K. et al. A UPF3-mediated regulatory switch that maintains RNA surveillance. Nat. Struct. Mol. Biol. 16, 747-753 (2009).

20. El-Bchiri, J. et al. Differential nonsense mediated decay of mutated mRNAs in mismatch repair deficient colorectal cancers. Hum. Mol. Genet. 14, 2435-2442 (2005).

21. El-Bchiri, J. et al. Nonsense-mediated mRNA decay impacts MSI-driven carcinogenesis and anti-tumor immunity in colorectal cancers. PLOS ONE 3, e2583 (2008).

22. Shum, E. Y. et al. The antagonistic gene paralogs Upf3a and Upf3b govern nonsense-mediated RNA decay. Cell 165, 382-395 (2016).

23. Lee, J. H. et al. Frameshift mutation of WISP3 gene and its regional heterogeneity in gastric and colorectal cancers. Hum. Pathol. 50, 146-152 (2016).

24. Bohlig, L., Friedrich, M. \& Engeland, K. p53 activates the PANK1/miRNA-107 gene leading to downregulation of CDK6 and p130 cell cycle proteins. Nucleic Acids Res. 39, 440-453 (2011).

25. Lin, J. L., Liu, F. H. \& Wang, C. Interaction of Hsp70 with p49/STRAP, a serum response factor binding protein. Biochem. Biophys. Res. Commun. 389 607-611 (2009)

26. Chang, Y. J., Holtzman, M. J. \& Chen, C. C. Differential role of Janus family kinases (JAKS) in interferon-gamma-induced lung epithelial ICAM-1 expression: involving protein interactions between JAKs, phospholipase Cgamma, c-Src, and STAT1. Mol. Pharmacol. 65, 589-598 (2004).

27. Krishnan, K. Pine, R. \& Krolewski, J. J. Kinase-deficient forms of Jak1 and Tyk2 inhibit interferon alpha signaling in a dominant manner. Eur. J. Biochem. 247, 298-305 (1997).

28. Collura, A. et al. Patients with colorectal tumors with microsatellite instability and large deletions in HSP110 T17 have improved response to 5-fluorouracilbased chemotherapy. Gastroenterology 146, 401-411 (2014).

29. Dorard, C. et al. Expression of a mutant HSP110 sensitizes colorectal cancer cells to chemotherapy and improves disease prognosis. Nat. Med. 17, 1283-1289 (2011)

30. Gonzalez-Hilarion, S. et al. Rescue of nonsense mutations by amlexanox in human cells. Orphanet J. Rare Dis. 7, 58 (2012).

31. Durand, S. et al. Inhibition of nonsense-mediated mRNA decay (NMD) by a new chemical molecule reveals the dynamic of NMD factors in P-bodies. J. Cell Biol. 178, 1145-1160 (2007).

32. Ionov, Y., Nowak, N., Perucho, M., Markowitz, S. \& Cowell, J. K. Manipulation of nonsense mediated decay identifies gene mutations in colon cancer cells with microsatellite instability. Oncogene 23, 639-645 (2004).

33. Ouyang, Z., Zheng, G., Tomchick, D. R., Luo, X. \& Yu, H. Structural basis and IP6 requirement for Pds5-dependent cohesin dynamics. Mol. Cell 62, 248-259 (2016). 
34. Berthenet, $\mathrm{K}$. et al. HSP110 promotes colorectal cancer growth through STAT3 activation. Oncogene 36, 2328-2336 (2016).

35. Yokoyama, T. et al. DA-Raf1, a competent intrinsic dominant-negative antagonist of the Ras-ERK pathway, is required for myogenic differentiation. J. Cell Biol. 177, 781-793 (2007).

36. Carter, M. S. et al. A regulatory mechanism that detects premature nonsense codons in T-cell receptor transcripts in vivo is reversed by protein synthesis inhibitors in vitro. J. Biol. Chem. 270, 28995-29003 (1995).

37. Wang, D. et al. Inhibition of nonsense-mediated RNA decay by the tumor microenvironment promotes tumorigenesis. Mol. Cell. Biol. 31, 3670-3680 (2011).

38. Noensie, E. N. \& Dietz, H. C. A strategy for disease gene identification through nonsense-mediated mRNA decay inhibition. Nat. Biotechnol. 19, 434-439 (2001).

39. Dang, $Y$. et al. Inhibition of nonsense-mediated mRNA decay by the natural product pateamine A through eukaryotic initiation factor 4All. J. Biol. Chem. 284, 23613-23621 (2009).

40. Yamashita, A., Ohnishi, T., Kashima, I., Taya, Y. \& Ohno, S. Human SMG-1, a novel phosphatidylinositol 3-kinase-related protein kinase, associates with components of the mRNA surveillance complex and is involved in the regulation of nonsense-mediated mRNA decay. Genes Dev. 15, 2215-2228 (2001).

41. Ballal, V. V. J. Oral medicine: Amlexanox. Br. Dent. J. 217, 208 (2014).

42. Belenguer-Guallar, I., Jimenez-Soriano, Y. \& Claramunt-Lozano, A. Treatment of recurrent aphthous stomatitis. A literature review. J. Clin. Exp. Dent. 6 e168-e174 (2014)

43. Reilly, S. M. et al. An inhibitor of the protein kinases TBK1 and IKK-varepsilon improves obesity-related metabolic dysfunctions in mice. Nat. Med. 19, 313-321 (2013).

44. Buhard, O. et al. Multipopulation analysis of polymorphisms in five mononucleotide repeats used to determine the microsatellite instability status of human tumors. J. Clin. Oncol. 24, 241-251 (2006).

45. Dorard, C. et al. Expression of a mutant HSP110 sensitizes colorectal cancer cells to chemotherapy and improves disease prognosis. Nat. Med. 17, 1283-1289 (2011).

46. Marisa, L. et al. Gene expression classification of colon cancer into molecular subtypes: characterization, validation, and prognostic value. PLoS Med. 10, e1001453 (2013). 